\title{
Immunohistochemical analysis of the expression of FATE/BJ-HCC-2 antigen in normal and malignant tissues
}

\author{
Xiao Ang Yang ${ }^{1}$, Xue Yuan Dong ${ }^{1}$, Huan Qiao ${ }^{1}$, Yue Dan Wang ${ }^{1}$, Ji Run Peng ${ }^{2}$, Yan Li $^{1}$, \\ Xue Wen Pang ${ }^{1}$, Chan Tian ${ }^{1}$ and Wei Feng Chen ${ }^{1}$ \\ ${ }^{1}$ Department of Immunology, School of Basic Medical Sciences, Peking University Health Science Center, \\ Beijing, China and ${ }^{2}$ Center of Hepatobiliary Surgery, People's Hospital, Peking University Health Science \\ Center, Beijing, China
}

\begin{abstract}
FATE/BJ-HCC-2 is a newly identified cancer/testis (CT) antigen, which was detected in tumor tissues and testis. As previous studies of $F A T E / B J-H C C-2$ expression pattern were mainly based on messenger RNA (mRNA) analysis, it is necessary to investigate its actual protein expression pattern in tumor tissues for the evaluation of its application value. In this study, we produced specific polyclonal antibody (pAb) to the recombinant FATE/ BJ-HCC-2 protein and analyzed the FATE/BJ-HCC-2 antigen expression in normal and malignant tissues by the immunohistochemical approach. The results showed that there was no detectable FATE/BJ-HCC-2 antigen expressed in normal tissues except testis. In hepatocellular carcinoma (HCC) tissues, the FATE/BJ-HCC-2 antigen was detected in $20 \%$ (7/35) specimens. All samples that expressed the FATE/BJ-HCC-2 antigen were of poorly or moderately differentiated HCC. The stained antigen was located in the cytoplasm and the staining pattern showed heterogeneity from focal to more than $40 \%$ of the tumor cells. The FATE/BJ-HCC-2 antigen was also expressed in other tumor tissues. The results of $\left[{ }^{3} \mathrm{H}\right]$ thymidine incorporation showed that FATE/BJ-HCC-2 protein enhanced tumor cell proliferation after transfection of $F A T E / B J-H C C-2$ gene in HCC cell line $(P<0.01)$. This effect could be specifically blocked by anti-FATE/BJ-HCC-2 pAb. Serological screening showed that the antibody specific to the FATE/BJ-HCC-2 antigen was detected in $7.7 \%(4 / 52)$ patients. Notably, the four positive patients bore poorly or moderately differentiated HCC. FATE/BJ-HCC-2 mRNA transcript was detected in the peripheral blood mononuclear cells (PBMCs) of $46.67 \%$ patients whose resected HCC tissue samples were positive for FATE/BJ-HCC-2 mRNA, which implicated tumor cell dissemination in blood circulation and may relate to the metastasis of HCC. Thus, FATE/BJ-HCC-2 may be a valuable candidate CT antigen for polyvalent vaccines in tumor immunotherapy and an assisting diagnostic marker for prognosis of the disease.

Laboratory Investigation (2005) 85, 205-213, advance online publication, 6 December 2004; doi:10.1038/labinvest.3700220
\end{abstract}

Keywords: cancer/testis antigen; FATE/BJ-HCC-2; FATE/BJ-HCC-2 antigen; hepatocellular carcinoma; immunohistochemical analysis

Successful immunotherapy of cancer patients requires the recognition of tumor-associated antigens by the immune system. To identify the tumorassociated antigens capable of eliciting immune responses in autologous host is a major aim of tumor immunology. ${ }^{1,2}$ Taking the advent of the discovery of a large number of tumor-associated antigens over the last decade, the tumor immunotherapy with various strategies has been approached

Correspondence: Professor WF Chen, MD, PhD, Department of Immunology, School of Basic Medical Sciences, Peking University Health Science Center, 38 Xue Yuan Road, Beijing 100083, China.

E-mail: wfchen@public.bta.net.cn

Received 18 May 2004; revised and accepted 25 October 2004; published online 6 December 2004 using the identified genes and their encoding proteins. Cancer/testis (CT) antigens are a category of tumor antigens with normal expression restricted to male germ cells in the testis but not in other adult somatic tissues. In malignancy, these genes are demethylated and activated, resulting in CT antigen expression in a proportion of tumors of various histological types. Since their initial identification by T-cell epitope cloning, the list of CT antigens has been greatly expanded through serological analysis of recombinant cDNA expression libraries (SEREX) and differential messenger RNA (mRNA) expression analysis. ${ }^{3-7}$ Most CT antigen genes are localized on the X-chromosome, often clustered as multigene families. These antigens generally elicit spontaneous humoral and cell-mediated immune responses in tumor patients. CT antigens appear to be the ideal 
tumor-specific antigen in tumor immunotherapy because the spermatogenic cells do not express HLA molecules and are unable to induce autoimmune response in testis. ${ }^{2}$

FATE/BJ-HCC-2 was originally identified as a testis-specific gene and we have recently identified it as a CT antigen-encoding gene, which maps to chromosome Xq28, ${ }^{8,9}$ According to the late classification of CT antigens, the FATE/BJ-HCC-2 is nominated as FATE/CT43. ${ }^{7}$ We have demonstrated that FATE/BJ-HCC-2 mRNA is highly expressed in hepatocellular carcinoma (HCC) and not in normal tissues except testis by RT-PCR. ${ }^{8}$ However, we do not know the extent of FATE/BJ-HCC-2 protein expression, an essential condition for the antigen to elicit immune response in HCC and in other neoplastic tissues. In this study, we have evaluated the FATE/BJ-HCC-2 antigen expression in normal and malignant tissues by immunohistochemical (IHC) assay with a polyclonal antibody (pAb) specific to the recombinant FATE/BJ-HCC-2 protein (anti-FATE/BJ-HCC-2 pAb). In addition, a parallel comparison was made between the expression of the mRNA transcript and the protein of FATE/BJ-HCC-2 in some HCC specimens. The seroreactivity of HCC patients to the recombinant FATE/BJ-HCC-2 protein was screened by Western blotting assay. The correlation between the frequency of FATE/BJ-HCC-2 protein expression in HCC and the differentiation states of HCC was analyzed. We also observed that the FATE/BJ-HCC-2 gene product enhanced cell proliferation in the transfectant HCC cell line (Bel7402 cell) and detected the FATE/BJ-HCC-2 gene transcript in peripheral blood mononuclear cells (PBMCs) of HCC patients, and analyzed its potential clinical value.

\section{Materials and methods}

\section{Preparation of the Recombinant FATE/BJ-HCC-2 Protein}

The recombinant FATE/BJ-HCC-2 protein was expressed in prokaryotic system, which was prepared as in the previous report. ${ }^{10}$

\section{Production of the pAb to the Recombinant FATE/BJ-HCC-2 Protein}

Rabbits were immunized four times subcutaneously at 3-week intervals, with $200 \mu \mathrm{g}$ purified recombinant FATE/BJ-HCC-2 protein in adjuvant for every rabbit. Complete Freund's adjuvant was used for the first-time immunization; in the subsequent immunizations, incomplete Freund's adjuvant (Sigma, St Louis, MO, USA) was used. After the fourth time, the rabbit antisera were tested by solid-phase enzyme-linked immunosorbent assay (ELISA) on microtiter plates coated with the immunizing recombinant FATE/BJ-HCC-2 protein as a target anti- gen. Titer of antiserum was over 1:20 000. Lysates of Escherichia coli were used to absorb nonspecific antibodies against the residual E. coli components in the rabbit antisera. The anti-FATE/BJ-HCC-2 pAb was purified by a protein A column using the instructions of the manufacturer (Pierce, Rockford, IL, USA). The purified antibody was also absorbed with the FATE/BJ-HCC-2 protein to estimate its specificity in IHC experiments. The final concentration of the pAb for IHC staining was $0.5 \mu \mathrm{g} / \mathrm{ml}$.

\section{Tissue Sections, Sera and PBMC Samples}

Tissues sections were provided by the Departments of Pathology of Peking University Health Science Center and the People's Hospital of Peking University. The specimens consisted of formalin-fixed, paraffin-embedded tissue blocks and O.C.T.-embedded (TissueTek, Torrance, CA, USA) snap-frozen tissue samples. In all, 5- $\mu \mathrm{m}$ cuts were made from paraffin or frozen blocks and applied to histology slides for IHC staining. Paraffin sections were dried for $1 \mathrm{~h}$ at $80^{\circ} \mathrm{C}$. A panel of normal and neoplastic tissues was tested as indicated in Tables 1-3. Since paraffin-embedded specimens represent the standard archival material readily available for antigen expression studies, our analysis concentrated on formalin-fixed, paraffin-embedded tissues. However, for specificity analysis, partial HCC tissues were frozen specimens used for immunohistochemistry and mRNA detection.

The sera and the PBMC samples of HCC patients were from the People's Hospital of Peking University. The sera in this study were not of the same batch as in our previous studies of Dong et al. ${ }^{8}$ Patients agreed to the collection of blood and tissue samples with written consent and this was permitted by the Hospital Ethic Review Committee.

\section{IHC Staining}

Sections were deparaffinized and rehydrated with xylene and a series of grades of alcohol. Epitope retrieval was carried out in $10 \mathrm{mM}$ citrate buffer $(\mathrm{pH}$ 6.0) or $1 \mathrm{mM}$ EDTA buffer (pH 8.0) for $15 \mathrm{~min}$ at $95^{\circ} \mathrm{C}$ in a microwave cooker, followed by cooling for $30 \mathrm{~min}$ at room temperature. After inactivation of endogenous peroxidase with $\mathrm{H}_{2} \mathrm{O}_{2}$, sections were blocked with $10 \%$ normal goat serum for $30 \mathrm{~min}$ and then incubated with the anti-FATE/BJ-HCC-2 pAb at room temperature for $1 \mathrm{~h}$. After washing, horseradish peroxidase (HRP)-conjugated anti-rabbit IgG (Zymed laboratories, Inc., South San Francisco, CA, USA) was added and further incubated for $20 \mathrm{~min}$ at room temperature. The slides were washed, and diaminobenzidine tetrahydrochloride was then added as a chromogen (Zhongshan Biotech Corp., Beijing), followed by counterstaining with hematoxylin solution. Sections of normal human testis were used as positive control. An unrelated rabbit IgG at the 
same concentration was used as a negative control. The anti-FATE/BJ-HCC-2 pAb after neutralization with the FATE/BJ-HCC-2 protein served as another control to confirm the specificity of staining. IHC staining of positive samples was repeated twice.

\section{RT-PCR}

To determine the specificity of the anti-FATE/ BJ-HCC-2 pAb, RT-PCR analysis and IHC staining of the FATE/BJ-HCC-2 protein were compared in partial HCC samples. RT-PCR was carried out as previously described. ${ }^{8}$ Briefly, total RNA was extracted from frozen tissue samples of HCC. Testicular tissue was used as a positive control tissue. RNA was reversely transcribed with AMV reverse transcriptase and oligo dT(18) (Clontech, Palo Alto, CA, USA). The cDNA from testis was used as a positive control. The integrity and quality of the cDNA were confirmed by PCR for G3PDH. Gene-specific PCR primers used to amplify FATE/BJ-HCC-2 were designed according to its sequences (forward: ctg ttc ctg gca ccc tgt gca tcc; reverse: gat gcc gcc atg ctg ttc acc c). RT-PCR was performed with the Advantage Taq DNA polymerase (Clontech) for 30 cycles in GeneAmp PCR system 9700 (Applied Biosystems, Foster, CA, USA) at an annealing temperature of $66^{\circ} \mathrm{C}$. Products were analyzed on $1.2 \%$ agarose gels containing $0.01 \mathrm{mg} / \mathrm{ml}$ ethidium-bromide. The relative quantity of RT-PCR products of mRNA in tumor tissues was assessed by a Bio-Rad Quantity One-4.3.0 analytic system.

For detecting FATE/BJ-HCC-2 gene transcript in PBMCs, total RNA was extracted from freshly isolated PBMCs using TRIzol reagent according to the manufacturer's instructions (Invitrogen, Carlsbad, CA, USA). The cDNA synthesis from total RNA was the same as that described above. The nested PCR amplifications were performed under the following conditions: (1) The cDNA samples from PBMCs were denatured at $94^{\circ} \mathrm{C}$ for $3 \mathrm{~min}$ followed by $94^{\circ} \mathrm{C}$ for $30 \mathrm{~s}, 63^{\circ} \mathrm{C}$ for $30 \mathrm{~s}$, and $72^{\circ} \mathrm{C}$ for $30 \mathrm{~s}$ for 30 cycles. The primers were as follows: forward, 5'-AGG AGG CCC TCC CAA CAC CAA G-3' and reverse, 5'-GCA CGG CGA TGA TCA GGG TCTC-3', (2) For the second round of PCR, 2.5 $\mu$ l of a 1:10 dilution of the first-round PCR product was used as template to amplify FATE/BJ-HCC-2 cDNA. The inner primers were: forward, 5'-CGG AGA TGG AAA TGT CCC TGG CAG-3'; reverse, 5'-GAC GCC GGT TGA CTG CAT ACA GC-3', which were designed with Gene Runner analysis software 3.04 (Hastings Software Inc.). After initial heating at $94^{\circ} \mathrm{C}$ for $3 \mathrm{~min}$, 30 cycles of PCR were carried out for the amplification $\left(94^{\circ} \mathrm{C}\right.$ for $30 \mathrm{~s}, 65^{\circ} \mathrm{C}$ for $30 \mathrm{~s}$, and $72^{\circ} \mathrm{C}$ for $30 \mathrm{~s})$.

\section{Transfection of FATE/BJ-HCC-2 Gene in HCC Cell Line}

The HCC cell line (Bel-7402 cell) was maintained in our laboratory. The pcDNA3 plasmid was from the
Life Science Institute of Peking University. The pcDNA3-FATE/BJ-HCC-2 expression plasmid was constructed for cell transfection. The open reading frame of FATE/BJ-HCC-2 was obtained by PCR strategy (sense: 5'-CG CGG ATC CTG GTT CAT CCA CAG CCA CAG-3', antisense: 5'-CCC AAG CTT GCC ACC ATG GCA GGA GGC CCT CCC AA-3') and was inserted into the plasmid pcDNA3, with a Carboxy (C)-terminal FLAG tag. The expression plasmid was transfected into Bel-7402 cells using lipofectamine 2000 reagent according to the manufacturer's instructions (Invitrogen). The Bel-7402 cells transfected with pcDNA3 vector were simultaneously produced as mock control. The Bel-7402 cells transfected with pcDNA3-FATE/BJ-HCC-2 expression plasmid expressed the target protein verified by immunofluorescence assay. For stable transfection, the transfectant cells were selected by adding the antibiotic G418 for 3 weeks to kill the cells without pcDNA3-FATE/BJ-HCC-2 expression plasmids.

\section{Transfer of Antibody into Transfectant Cells by Electroporation}

The stably transfected Bel-7402 and mock control cells were digested and washed twice with serumfree medium. In all, $6 \times 10^{5}$ cells were incubated with $1 \mathrm{mg} / \mathrm{ml}$ anti-FATE/BJ-HCC-2 pAb in $0.6 \mathrm{ml}$ serum-free medium for 10 min on ice. Then the cells in $0.2 \mathrm{~cm}$ Bio-Rad electrode gap cuvettes were exposed to a single brief electric pulse of $120 \mathrm{~V}$ and a duration of $20 \mathrm{~ms}$ delivered from Bio-Rad Gene Pulser (Bio-Rad Laboratories, Hercules, CA, USA) at room temperature.

\section{$\left[{ }^{3} \mathrm{H}\right]$ thymidine Incorporation}

The FATE/BJ-HCC-2 gene-transfected Bel-7402 and mock control cells with or without pAb transfer were incubated in 96-well flat-bottom culture plates (Costar, Corning, NY, USA) at a density of $2 \times 10^{4}$ cells/well. $\left[{ }^{3} \mathrm{H}\right]$ thymidine (Beijing Atomic Energy Institute, China) of $0.5 \mu \mathrm{Ci}(18.5 \mathrm{kBq}) /$ well was added $12 \mathrm{~h}$ before harvest. After being cultured for $24 \mathrm{~h}$, the cells were collected with Harvester 96 Mach II (Tomtec Inc., CT, USA) and CPM value for each well was determined in scintillation fluid on $\beta$ counter (PerkinElmer Life and Analytical Sciences, MA, USA).

\section{Statistical Analysis}

The results were expressed as mean \pm standard error of the mean. The statistical significance was determined by applying a two-tailed Student's $t$-test. $p$-values $<0.05$ were considered as statistically significant. 


\section{Western Blotting}

The humoral immune response against the FATE/ BJ-HCC-2 antigen in HCC patients was screened by Western blotting assay. Briefly, the purified FATE/ BJ-HCC-2 protein was separated on $10 \%$ SDSpolyacrylamide gel through electrophoresis and transferred onto nitrocellulose membranes. After blocking with $5 \%$ skim milk in the TNT buffer (20 mM Tris-HCl, $100 \mathrm{mM} \mathrm{NaCl,} 0.05 \%$ Tween 20, $\mathrm{pH}$ 7.5), the membranes were sequentially incubated with HCC patients' sera of a 1:500 dilution for $1 \mathrm{~h}$, then incubated with goat anti-human $\operatorname{IgG}(\mathrm{H}+\mathrm{L})$ alkaline phosphatase conjugate (Promega corporation, Madison, WI, USA) for $1 \mathrm{~h}$, and color substrate NBT/BCIP (Promega) was added for color development. To test the specificity of antibody response against the FATE/BJ-HCC-2 protein, the irrelevant protein expressed in E. coli was applied as control in Western blot assay, in which the antibody was only detected against FATE/BJ-HCC-2, but not to irrelevant protein.

\section{Results}

\section{Distribution of the FATE/BJ-HCC-2 Antigen in Normal Tissues}

Table 1 summarizes the IHC staining properties of the anti-FATE/BJ-HCC-2 pAb for normal tissues. Testis was the only normal tissue immunoreactive to the pAb, with germ cells and Sertoli cells in the seminiferous tubules showing strong staining. No staining was present in the interstitial tissue (Figure 1a). In the seminiferous tubules, the staining was most intense in spermatogonia and in primary spermatocytes; however, at the later stages of maturation, the staining intensity of spermatocytes became weak gradually, and matured spermatids were negative. The staining pattern of the FATE/BJHCC-2 antigen was cytoplasmic. Sertoli cells were also strongly immunoreactive (Figure 1a). No positive staining was found in ovary tissues and other

Table 1 FATE/BJ-HCC-2 antigen expression in normal tissues detected by IHC staining

\begin{tabular}{lc}
\hline Tissue samples & IHC staining \\
\hline Brain & - \\
Heart & - \\
Lung & - \\
Liver & - \\
Kidney & - \\
Stomach & - \\
Skeletal muscle & - \\
Pancreas & - \\
Small intestine & - \\
Ovary & - \\
Spleen & - \\
Lymph node & - \\
Prostate & - \\
Testis & +++ \\
\hline
\end{tabular}

normal tissue samples. However, in the paraffin sections of the pancreas, the Langerhans' cell islets were faintly stained when citrate was used as the antigen retrieval solution. This immunoreactivity of nontesticular normal tissues was absent when EDTA was employed as the antigen retrieval solution, while the staining of testicular tissue stably existed. Hence, EDTA should be used as the antigen retrieval solution in the staining of paraffin sections to reduce or remove nonspecific reactivity. ${ }^{11}$

\section{IHC Analysis in HCC}

The expression of the FATE/BJ-HCC-2 antigen in 35 HCC samples is summarized in Table 2. The overall positive rate of FATE/BJ-HCC-2 antigen expression was $20 \%$ (seven of 35 ). Notably, the proportion of FATE/BJ-HCC-2 antigen expression was reversely correlated with the HCC differentiated states. Higher frequency of FATE/BJ-HCC-2 antigen expression was seen in poorly or moderately differentiated HCC. The positive rate was $30.8 \%$ (four of 13 ) in moderately differentiated HCC and $25.0 \%$ (three of 12 ) in the poorly differentiated HCC, but there was no antigen expression to be detected (0/10) in welldifferentiated HCC. Among different HCC samples, only the cancer cells were observed to be positive by IHC staining with the anti-FATE/BJ-HCC-2 pAb. The staining intensity varied from weak to strong, and the staining pattern showed a high heterogeneity from focal to more than $40 \%$ of the cancer cells (Figure $2 b$ and c). The antigen was located in cytoplasm shown by IHC assay. To verify the specificity of IHC staining, sections cut from FATE/BJ-HCC-2-positive samples were stained with the FATE/BJ-HCC-2 proteinabsorbed anti-FATE/BJ-HCC-2 pAb, and exhibited IHC negative. Thus, the positive IHC staining specifically manifested the expression of the FATE/ BJ-HCC-2 antigen in HCC samples.

\section{Correlativity of the Expression of the FATE/BJ-HCC-2 Protein and mRNA in HCC Samples}

To further confirm the specificity of the anti-FATE/ BJ-HCC-2 antibody, the expression of the FATE/ BJ-HCC-2 protein and mRNA in HCC samples was compared. The FATE/BJ-HCC-2 mRNA expression was detected in $66 \%$ tumor samples as mentioned in our previous report. ${ }^{8}$ In this study, 15 HCC samples were available for both mRNA and protein analysis in parallel. Of these, three samples were both FATE/ BJ-HCC-2 protein positive as displayed by IHC staining and mRNA positive assessed by RT-PCR; seven samples were mRNA positive but protein negative in IHC staining; all these seven samples exhibited lower copies of mRNA shown in the relative quantitative analysis of PCR products (Figure $2 \mathrm{a}$ and $\mathrm{b}$ ); five samples were negative for both protein and mRNA. None of the FATE/BJ-HCC-2 mRNAnegative samples expressed the FATE/BJ-HCC-2 

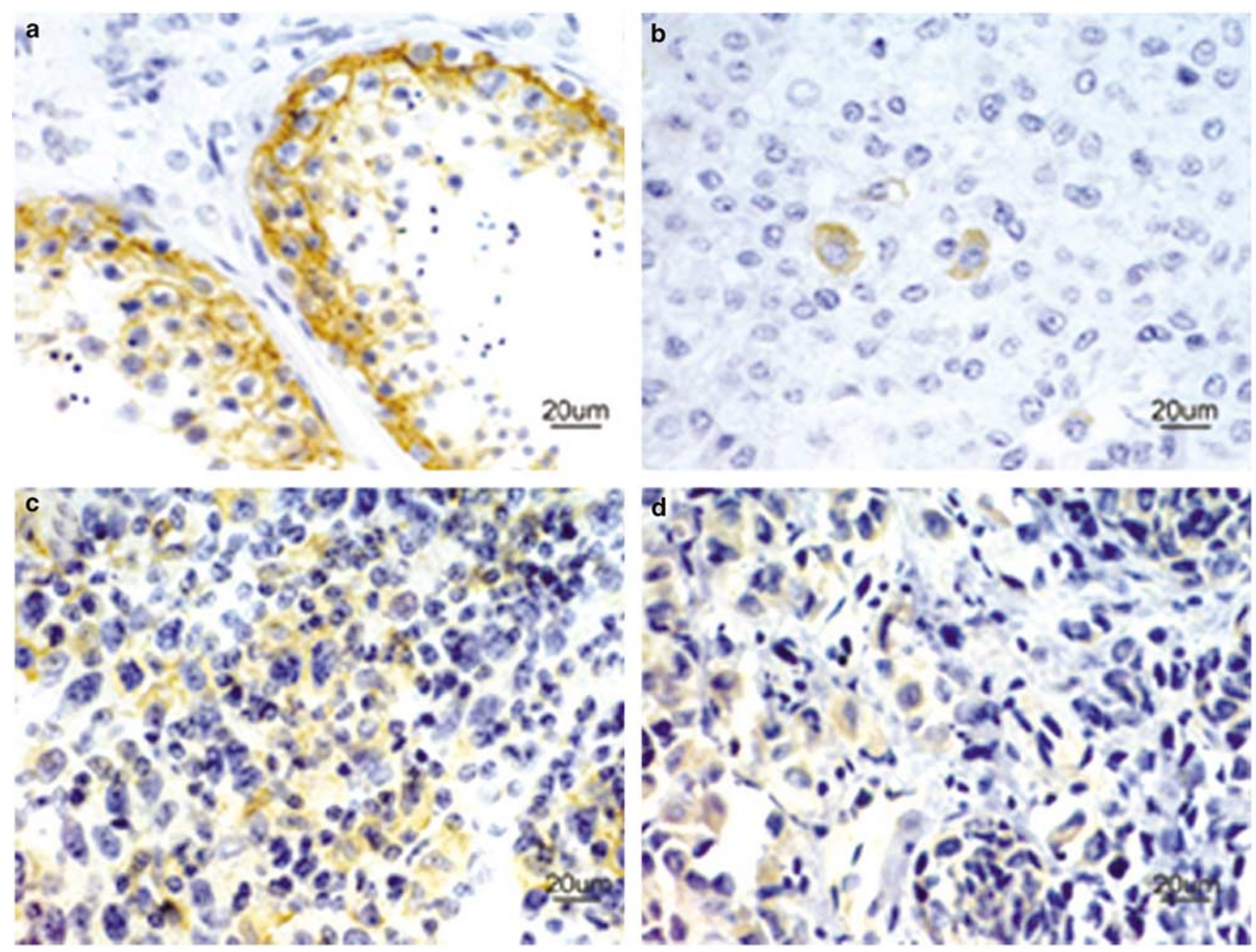

Figure 1 Immunohistochemical analysis of FATE/BJ-HCC-2 antigen expression in normal testis and malignant tumor tissues. The sections of tissue samples were stained with the anti-FATE/BJ-HCC-2 pAb followed by staining with peroxidase-conjugated anti-rabbit IgG $(\mathrm{H}+\mathrm{L})$. The color was developed with DAB chromogen and the sections were counterstained with hematoxylin. Original magnification $\times 400$. (a) In testis, the intensive IHC staining was shown in seminiferous tubules. Both germ cells and Sertoli cells were immunopositive. The antigen was expressed in cytoplasm. The intensity of the antigen expression was gradually weaker following spermatogonia maturing process. The spermatids were negative. There was no staining in interstitial tissue of testis. (b, c) Heterogeneous expression of the FATE/BJ-HCC-2 antigen in HCC tissues. (b) Focal immunostaining of tumor cells in moderately differentiated HCC. (c) Cluster immunostaining of tumor cells in poorly differentiated HCC. (d) Immunopositive tumor cells in malignant melanoma.

Table 2 FATE/BJ-HCC-2 antigen expression in HCC tissues detected by IHC staining

Grades Samples tested Positive number Positive rate (\%)

\begin{tabular}{lccc}
\hline Well & 10 & 0 & 0 \\
Moderate & 13 & 4 & 30.8 \\
Poor & 12 & 3 & 25.0 \\
Total & 35 & 7 & 20.0 \\
\hline
\end{tabular}

protein, indicating the specificity of IHC staining in HCC with the anti-FATE/BJ-HCC-2 pAb.

\section{Immunohistochemical Analysis of FATE/BJ-HCC-2 Antigen Expression in Other Malignant Tumors}

Table 3 shows the expression of the FATE/BJ-HCC-2 antigen in a panel of tumors except HCC. The FATE/
BJ-HCC-2 antigen was positive in melanoma (2/8) and breast cancer (1/10) samples. The anti-FATE/ BJ-HCC-2 pAb reactive cell was not seen in renal cell carcinomas, lymphoma, colorectal cancers, gastric cancers and other tumors (Table 3).

\section{Proliferation Assay of FATE/BJ-HCC-2 Gene-Transfected Bel 7402 Cells}

To identify whether the FATE/BJ-HCC-2 antigens play a role in tumor cell proliferation, DNA synthesis in the FATE/BJ-HCC-2 gene-transfected Bel-7402 cells was estimated by $\left[{ }^{3} \mathrm{H}\right]$ thymidine incorporation. The result showed that the uptake amount of $\left[{ }^{3} \mathrm{H}\right]$ thymidine was significantly increased in the FATE/BJ-HCC-2 gene-transfected Bel-7402 cells compared with that of the mock control (202 274.0 \pm 20078.4 vs $154665.2 \pm 12473.2$, $P<0.01$ ). To block the role of FATE/BJ-HCC-2 protein 

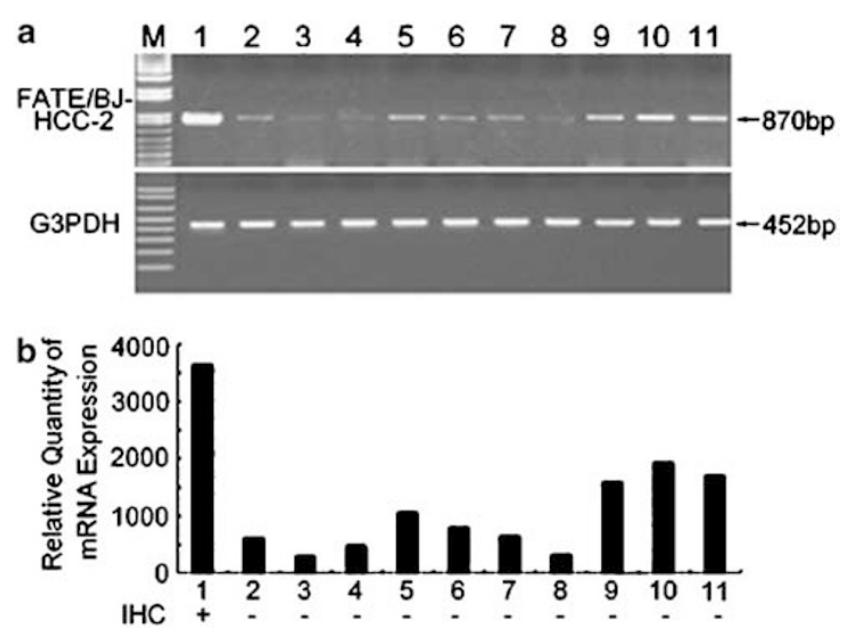

Figure 2 Comparison of the FATE/BJ-HCC-2 mRNA expression level and the respective protein expression. The FATE/BJ-HCC-2 mRNA in HCC tissue specimens was assessed by RT-PCR and the expressed mRNA was semiquantitated by density scanning. (a) Agarose gels electrophoresis analysis of RT-PCR products. The G3PDH expression was applied as internal control to normalize the RNA amount among the samples. (b) Relative quantity of RTPCR products. The bottom of the figure shows the IHC results in the corresponding HCC tissue samples. M, DNA marker; Lane 1, mRNA expression of testis as a positive control; Lanes 2-8, mRNA expression in HCC samples in which FATE/BJ-HCC-2 protein was not detected by IHC staining; Lanes 9-11, mRNA expression in HCC samples in which FATE/BJ-HCC-2 protein was positive.

Table 3 FATE/BJ-HCC-2 antigen expression in other tumors detected by IHC staining

\begin{tabular}{lrc}
\hline Tumors & Samples tested & Positive number \\
\hline Melanoma & 8 & 2 \\
Breast cancer & 10 & 1 \\
Renal cell cancer & 3 & 0 \\
Lymphoma & 8 & 0 \\
Gastric cancer & 9 & 0 \\
Colorectal cancer & 15 & 0 \\
Prostate cancer & 5 & 0 \\
Lung cancer & 4 & 0 \\
Pancreas cancer & 8 & 0 \\
Neurinoma & 2 & 0 \\
Bladder cancer & 9 & 0 \\
\hline
\end{tabular}

in the promotion of cell proliferation, the FATE/BJHCC-2 pAb was transferred into the transfectant Bel7402 cells. The uptake amount of $\left[{ }^{3} \mathrm{H}\right]$ thymidine was significantly reduced in the FATE/BJ-HCC-2 genetransfected Bel-7402 cells after anti-FATE/BJ-HCC-2 $\mathrm{pAb}$ was electroporated into the transfectant cells (162 230.4 \pm 6850.8 vs $202274.0 \pm 20078.4, P<0.01$ ). In contrast, there was no significant difference between the mock controls with and without $\mathrm{pAb}$ transferred into the cells (143402.8 \pm 4549.401 vs $154665.2 \pm 12$ 473.16, $P>0.05$ ) (Figure 3).

\section{FATE/BJ-HCC-2 mRNA Expression in PBMCs}

With nested PCR amplification, the positive rate of FATE/BJ-HCC-2 mRNA transcript in PBMCs of the

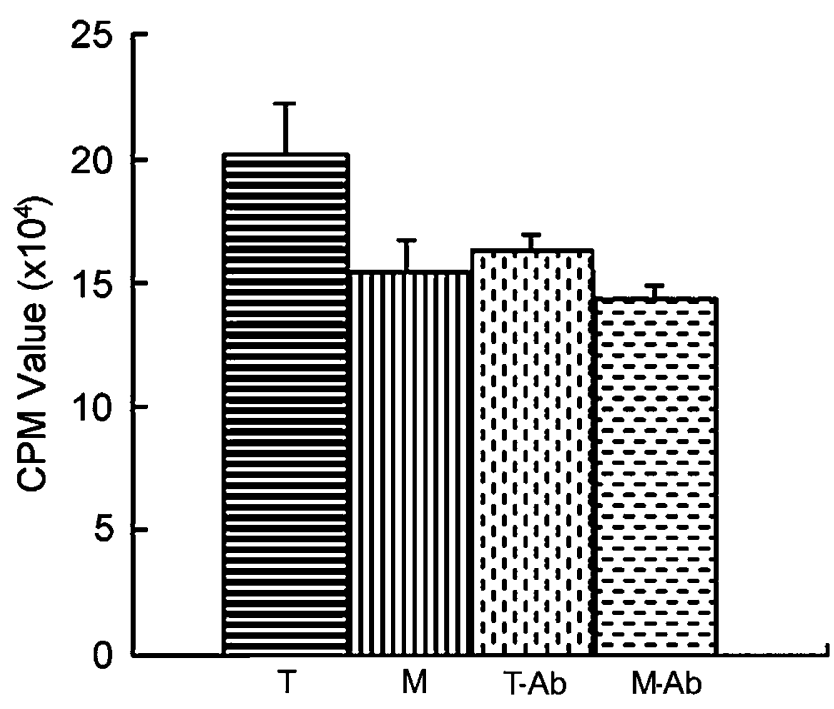

Figure 3 Cell proliferation and antibody blocking assays by $\left[{ }^{3} \mathrm{H}\right]$ thymidine incorporation in Bel-7402 cells. The FATE/ BJ-HCC-2 gene-transfected Bel-7402 and mock control cells with or without pAb electroporated were incubated in 96-well flatbottom culture plates. $\left[{ }^{3} \mathrm{H}\right]$ thymidine of $0.5 \mu \mathrm{Ci}(18.5 \mathrm{kBq}) /$ well was added into culture medium $12 \mathrm{~h}$ before harvest. The CPM value for each well was determined in scintillation fluid on $\beta$ counter. T, FATE/BJ-HCC-2 gene-transfected Bel-7402 cells; M, mock control; T-Ab, FATE/BJ-HCC-2 gene-transfected cells with anti-FATE/BJ-HCC-2 pAb blocking; M-Ab, mock control cells with anti-FATE/BJ-HCC-2 pAb blocking.

patients whose resected HCC tissue samples were positive for FATE/BJ-HCC-2 mRNA was $46.67 \%$ (Table 4). FATE/BJ-HCC-2 mRNA transcript was not detected in the PBMCs of the patients whose resected HCC tissue samples were negative for FATE/BJ-HCC-2 mRNA, or in the PBMC of normal donors. In light of the clinical data of the seven positive samples, four patients were classified as being in clinical phase III and three patients were in clinical phase I-II. Of the patients who were followed up, one patient whose PBMCs were positive for FATE/BJ-HCC-2 mRNA transcript, relapsed with metastasis of HCC within 6 months. In comparison, two patients whose PBMCs were negative for FATE/BJ-HCC-2 mRNA transcript did not relapse in 6 months.

\section{Seroreactivity Analysis of Antibody against the FATE/BJ-HCC-2 Protein in HCC Patients}

In the 52 sera collected from HCC patients, there were four reactive to the FATE/BJ-HCC-2 protein assessed by Western blotting (Figure 4). The experiment was repeated twice and the same results were obtained. Thus, the positive rate was $7.7 \%$. As the specific control of seroreactivity, the patient's sera, which were sero-positive to the FATE/BJ-HCC-2 protein, could not react with irrelevant protein. Of note, all these four sero-positive patients had the FATE/BJ-HCC-2 antigen expression in their resected 
Table 4 FATE/BJ-HCC-2 mRNA transcript in the various PBMC samples

\begin{tabular}{lccrc}
\hline Samples & $\begin{array}{c}\text { PBMC } \\
\text { positive }^{\mathrm{a}}\end{array}$ & $\begin{array}{c}\text { PBMC }_{\text {negative }} \\
\text { nestal }\end{array}$ & $\begin{array}{c}\text { Tositive } \\
\text { rate (\%) }\end{array}$ \\
\hline HCC tissue positive $^{\mathrm{a}}$ & 7 & 8 & 15 & 46.67 \\
HCC tissue negative $^{\mathrm{a}}$ & 0 & 5 & 5 & 0 \\
Normal PBMC & 0 & 5 & 5 & 0 \\
\hline
\end{tabular}

${ }^{\text {a }}$ Positive or negative: the samples were positive or negative for FATE/ HCC-2 mRNA.

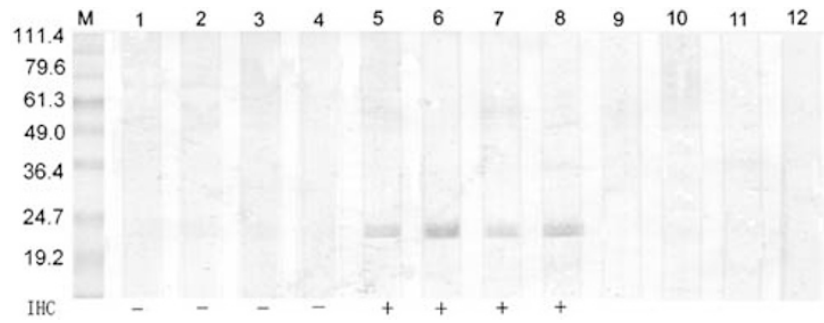

Figure 4 Western blot analysis of anti-FATE/BJ-HCC-2 antibody in sera of HCC patients. In Western blotting, the recombinant FATE/BJ-HCC-2 protein was used as antigen. M, protein marker; Lanes 1-4, anti-FATE/BJ-HCC-2 antibody was negative in patient's sera; Lanes 5-8, anti-FATE/BJ-HCC-2 antibody was positive in representative patient's sera. Lanes 9-12, the antiFATE/BJ-HCC-2 antibody positive sera did not immunoreact with irrelative protein. The bottom of the figure shows IHC results in the corresponding HCC tissue samples.

HCC tissues assessed by IHC analysis. In the analysis of the HCC differentiation states, all these four sero-positive HCC patients bore poorly or moderately differentiated HCC.

\section{Discussion}

CT antigens are the most attractive targets for vaccination in tumor immunotherapy, with the potential to eradicate residual tumor cells in multiple sites in cancer patients due to their expression in a variety of tumors but not in normal tissues except testis. ${ }^{3}$ In vitro and in vivo experiments have demonstrated that some CT antigens, for example, NY-ESO-1, are capable of provoking potent T-cellmediated immunity to directly kill tumor cells and release cytokines to interfere with the growth of tumor cells, and stimulated antibody response. ${ }^{12,13}$ Promising results have been observed in clinical trials using peptide vaccines derived from CT antigens for tumor immunotherapy. ${ }^{14,15}$

HCC is the third highest cause of cancer death worldwide with a global incidence of 1.2 million new cases per year. The recurrence of HCC after surgical resection is high to $70 \%$ and the prognosis of HCC remains poor. Despite many approaches of therapy, such as intra-arterial embolization, chemoembolization, regional chemotherapy, etc, there is no effective systemic therapy for this disease. ${ }^{16,17}$
Immunotherapy may be a promising strategy for HCC treatment. Identification of CT antigens from HCC is definitely needed to find more tumorassociated antigens with good immunogenicity for effective immunotherapy.

FATE/BJ-HCC-2 is a novel CT antigen and the previous analysis of its tissue expression pattern was mainly based on mRNA transcripts. ${ }^{7-9}$ Accurate information on the actual protein expression is a prerequisite for the evaluation of its immunogenicity. For this aim, we have prepared the recombinant FATE/BJ-HCC-2 protein and produced its specific pAb, so as to analyze the FATE/BJ-HCC-2 antigen expression in HCC and other tumor samples. The specificity of the anti-FATE/BJ-HCC-2 pAb was proved by three assays: (1) The pAb only reacted with the FATE/BJ-HCC-2 protein in immunoblotting, ${ }^{10}$ not with irrelevant protein. (2) The positive IHC staining was correlated with mRNA expression. (3) The pAb through preabsorption with purified FATE/BJ-HCC-2 protein no more gave IHC-positive staining in the samples, which were positive when stained with the pAb without absorption of specific antigen. With the confirmed specificity of the pAb, the results of IHC staining should be reliable. The FATE/BJ-HCC-2 antigen was expressed in substantial proportion in HCC samples $(20 \%, 7 / 35)$ and in melanoma $(25 \%, 2 / 8)$ as well as in low proportion in breast cancer $(10 \%, 1 / 10)$. The FATE/BJ-HCC-2 antigen was not expressed in colon, prostate and renal cell carcinomas, consistent with a low frequency of CT antigen expression in these tumors. ${ }^{6,18}$

Like other CT antigens, ${ }^{19-21}$ the FATE/BJ-HCC-2 antigen was highly expressed in the testicular tissue. Whereas most CT antigens are only restricted to germ cells, the FATE/BJ-HCC-2 antigen was expressed not only in spermatogonia and the primary spermatocytes but also in Sertoli cells of seminiferous tubule lumen. This may be associated with its function in both germ cells and Sertoli cells. Although we do not know the meaning of its expression in Sertoli cells, the expression of the FATE/BJ-HCC-2 antigen decreased following the maturation process of spermatogenic cells and virtually disappeared in the sperm cells. In addition, FATE/BJ-HCC-2 was coexpressed with SRY gene, which acts as a genetic switch that directs the development of the indifferent gonad from a female to a male pathway. ${ }^{9}$ The FATE/BJ-HCC-2 protein may hence function in early testicular differentiation. The expression of FATE/BJ-HCC-2 may also be involved in tumorigenesis as its expression is regulated by steroidogenic factor (SF-1) and Wilms' tumor gene 1 (WT1), the molecules involved in the development of tumors. ${ }^{22-24}$ The $\left[{ }^{3} \mathrm{H}\right]$ thymidine incorporation assay was used to measure cell DNA synthesis and hence cell proliferation. ${ }^{25}$ The results of $\left[{ }^{3} \mathrm{H}\right]$ thymidine incorporation assay in FATE/ $B J-H C C-2$ gene-transfected Bel-7402 cells showed that the FATE/BJ-HCC-2 protein enhanced tumor cell proliferation. This effect was specifically 
blocked by anti-FATE/BJ-HCC-2 pAb transferred into transfectant cells. Such an effect might be relevant to the advance of HCC as the FATE/BJ-HCC2 antigen was predominantly detected in lower differentiated HCC tissues. CT antigens were usually considered as targets of immunoresponses for immunotherapy and diagnostic markers of tumors. ${ }^{26,27}$ Of the 90 CT antigens identified at present, the biological function of the most of these antigens is unknown except SCP-1, OY-TES-1 and CT15/ Fertilin $\beta .^{3,7}$ Of course, whether the FATE/BJ-HCC-2 has other functions such as promoting tumor metastasis needs to be further investigated.

In the comparison of mRNA expression with protein expression in HCC samples, all the samples of the FATE/BJ-HCC-2 protein positive in IHC staining were also mRNA positive, detected by RTPCR. However, the protein expression was determined by the abundance of mRNA copies. In the relative quantitative analysis of RT-PCR products, the FATE/BJ-HCC-2 protein in samples expressed low abundance of mRNA and were unable to be detected by IHC staining.

There are two significant features revealed by IHC staining with the anti-FATE/BJ-HCC-2 pAb. First, the expression of the FATE/BJ-HCC-2 antigen correlated with the differentiation states of the HCC. This antigen is preferentially expressed in moderately and poorly differentiated HCC. Similar results were also shown in NY-ESO-1 expression in melanoma ${ }^{28}$ and MAGE-A4 in transitional cell carcinoma. ${ }^{29}$ Second, the IHC staining pattern is heterogeneous in different HCC samples, the expression of the FATE/BJ-HCC-2 antigen in tumor cells ranging from focal to a large mass of tumor cells. This heterogeneity appears to be a consistent morphologic characteristic of all CT antigens in many tumor types. ${ }^{19,21,30,31}$ This might be associated with spatial and temporal modulation of genes in the advanced tumors. ${ }^{32-34}$

Serological analysis of antibody to the FATE/ BJ-HCC-2 protein showed that $7.7 \%$ HCC patients developed spontaneous antibody responses without vaccine immunization. All these sero-positive HCC patients had the antigen expression in their resected HCC tissues. This suggests that the FATE/BJ-HCC-2 antigen is immunogenic as it elicited humoral immune responses in HCC patients. As the CT antigens can provoke $\mathrm{CD}^{+}$and $\mathrm{CD}^{+}$T-cell responses rather than induce antibody response, the availability of the recombinant protein makes us able to measure cellular response specific to this antigen through which to determine if it is a potential tumor antigen for clinical trial.

The high recurrence rate of HCC is probably attributed to the dissemination of tumor cells into blood circulation. Early detection of metastatic tumor cells is critical to identify HCC patients at high risk of relapse and for the prescriptive therapy. ${ }^{35}$ Since Smith et $a l^{36}$ first successfully adopted the RT-PCR technique to assess tyrosinase mRNA as a tumor marker to detect circulating melanoma cells, the gene transcripts of both tissueassociated and tumor-specific markers have been applied in RT-PCR-based diagnosis of micrometastasis of tumor cells in peripheral blood, such as the tyrosinase and $M A G E-3$ transcripts in melanoma, $M A G E-1$ and MAGE-3 in HCC, etc. ${ }^{35-39}$ The results in this study showed that the FATE/BJ-HCC-2 gene transcript was positive in PBMCs of $46.67 \%$ patients whose resected HCC tumor tissues were positive for FATE/BJ-HCC-2, implying the early dissemination of HCC cells in blood circulation. The FATE/BJ-HCC-2 gene transcript was detected in PBMCs in both the early and late clinical phase of disease. More importantly, one of those patients whose PBMCs sample was positive for FATE/BJ-HCC-2 mRNA, by the clinical data, relapsed with metastasis of HCC within 6 months. Two patients, whose FATE/ BJ-HCC-2 gene transcript was negative in PBMCs, did not relapse in 6 months. Thus, the persistent positivity for this gene transcript in PBMC may be a specific marker for poor prognosis of HCC patients. More patients need to be followed up for a longer term to confirm this phenomenon. Our previous reports also indicated that blood dissemination of tumor cells occurred in the early stage of HCC. ${ }^{35}$ Detection of the FATE/BJ-HCC-2 gene transcript in PBMCs by nested PCR may be useful for specifically diagnosing micrometastasis of HCC cells in peripheral blood and benefit the prediction of HCC recurrence and prognosis of the disease after surgical treatment.

In conclusion, we prepared the specific $\mathrm{pAb}$ to the FATE/BJ-HCC-2 antigen and demonstrated that this FATE/BJ-HCC-2 antigen expressed in HCC samples in around $20 \%$. This antigen can elicit humoral immune response in HCC patients that makes it a potential candidate to prepare tumor vaccine for immunotherapy of HCC patients provided that this antigen could also provoke CTL immune response. In view of the heterogeneous expression of CT antigens including the FATE/BJ-HCC-2 antigen in cancer tissues, it is necessary to develop polyvalent vaccines to prevent tumor cells escaping from immune attack.

\section{Acknowledgements}

We thank Professor WZ Zou and SL Liao for the help in pathological diagnosis. This work was supported by grants from the National 973 Program in China (No. G1999053904), Ludwig Institute for Cancer Research, New York (KSP \#003), and Beijing Municipal Government Foundation for Natural Sciences (No. 7001002).

\section{References}

1 Sahin U, Tureci O, Schmitt H, et al. Human neoplasms elicit multiple specific immune responses in the autologous host. Proc Natl Acad Sci USA 1995;92: 11810-11813. 
2 Park S, Lim Y, Lee D, et al. Identification and characterization of a novel cancer/testis antigen gene CAGE-1. Biochim Biophys Acta 2003;1625:173-182.

3 Scanlan MJ, Gure AO, Jungbluth AA, et al. Cancer/ testis antigens: an expanding family of targets for cancer immunotherapy. Immunol Rev 2002;188:22-32.

4 Juretic A, Spagnoli GC, Schultz-Thater E, et al. Cancer/ testis tumour-associated antigens: immunohistochemical detection with monoclonal antibodies. Lancet Oncol 2003;4:104-109.

5 Gure AO, Sahin U, Tsang S, et al. SSX: A multigene family with several members transcribed in normal testis and human cancer. Int J Cancer 1997;72:965-971.

6 Chen YT, Scanlan MJ, Sahin U, et al. A testicular antigen aberrantly expressed in human cancers detected by autologous antibody screening. Proc Natl Acad Sci USA 1997;94:1914-1918.

7 Scanlan MJ, Simpson AJG, Old LJ. The cancer/testis genes: review, standardization and commentary. Cancer Immunity 2004;4:1-15.

8 Dong XY, Su YR, Qian XP, et al. Identification of two novel CT antigens and their capacity to elicit antibody response in hepatocellular carcinoma patients. Br J Cancer 2003;89:291-297.

9 Olesen C, Larsen NJ, Byskov AG, et al. Human FATE is a novel X-linked gene expressed in fetal and adult testis. Mol Cell Endocrinol 2001;184:25-32.

10 Yang XA, Dong XY, Li Y, et al. Purification and refolding of a novel cancer/testis antigen FATE/ BJ-HCC-2 expressed in the inclusion bodies of Escherichia coli. Protein Exp Purif 2004;33:332-338.

11 Jungbluth AA, Chen YT, Busam KJ, et al. CT7 (MAGEC1) antigen expression in normal and neoplastic tissues. Int J Cancer 2002;99:839-845.

12 Jager D, Jager E, Knuth A. Immune responses to tumour antigens: implications for antigen specific immunotherapy of cancer. J Clin Pathol 2001;54:669-674.

13 Kirkin AF, Dzhandzhugazyan KN, Zeuthen J. Cancer/ testis antigens: structural and immunobiological properties. Cancer Invest 2002;20:222-236.

14 Marchand M, van Baren N, Weynants P, et al. Tumor regressions observed in patients with metastatic melanoma treated with an antigen peptide encoded by gene Mage-3 and presented by HLA-A 1 . Int J Cancer 1999;18:219-230.

15 Thurner B, Haendle I, Roder C, et al. Vaccination with Mage-3A1 peptide pulsed mature, monocyte-derived dendritic cells expands specific cytotoxic T cells and induced regression of some metastases in advanced stage IV melanoma. J Exp Med 1999;190:1669-1678.

16 Okuda K. Hepatocellular carcinoma. J Hepatol 2000; 32(Suppl 1):225-232.

17 Butterfield LH, Ribas A. Immunotherapy of hepatocellular carcinoma. Expert Opin Biol Ther 2002;2: 123-133.

18 Tureci O, Chen YT, Sahin U, et al. Expression of $S S X$ genes in human tumors. Int J Cancer 1998;77:19-23.

19 Jungbluth AA, Chen YT, Stockert E, et al. Immunohischemical analysis of NY-ESO-1 antigen expression in normal and malignant human tissues. Int J Cancer 2001;92:856-860.

20 Boon T, Old LJ. Cancer tumor antigens. Curr Opin Immunol 1997;19:681-683.

$21 \mathrm{Li} \mathrm{B}$, Qian XP, Pang XW, et al. HCA587 antigen expression in normal tissues and cancers: correlation with tumor differentiation in hepatocellular carcinoma. Lab Invest 2003;83:1185-1192.
22 Aylwin SJ, Welch JP, Davey CL, et al. The relationship between steroidogenic factor 1 and DAX-1 expression and in vitro gonadotropin secretion in human pituitary adenomas. J Clin Endocrinol Metab 2001;86: $2476-2483$.

23 Oka Y, Tsuboi A, Elisseeva OA, et al. WT1 as a novel target antigen for cancer immunotherapy. Curr Cancer Drug Targets 2002;2:45-54.

24 Sugiyama H. Wilms' tumor gene WT1: its oncogenic function and clinical application. Int J Hematol 2001; 73:177-187.

25 Mujoomder M, Bennett A, Hoskin D, et al. Adenosine stimulation of proliferation of breast carcinoma cell lines: evaluation of the $\left[{ }^{3} \mathrm{H}\right]$-thymidine assay system and modulatory effects of the cellular microenvironment in vitro. J Cell Physiol 2004;201:429-438.

26 Bodey B. Cancer-testis antigens: promising targets for antigen directed antineoplastic immunotherapy. Expert Opin Biol Ther 2002;2:577-584.

27 Hofmann M, Ruschenburg I. mRNA detection of tumor-rejection genes $B A G E, G A G E$, and $M A G E$ in peritoneal fluid from patients with ovarian carcinoma as a potential diagnostic tool. Cancer 2002;96:187-193.

28 Goydos JS, Patel M, Shih W. NY-ESO-1 and CTp11 expression may correlate with stage of progression in melanoma. J Surg Res 2001;98:76-80.

29 Kocher T, Zheng M, Bolli M, et al. Prognostic relevance of MAGE0A4 tumor antigen expression in transitional cell carcinoma of the urinary bladder: a tissue microarray study. Int J Cancer 2002;100:702-705.

30 Jungbluth AA, Stockert E, Chen YT, et al. Monoclonal antibody MA454 reveals a heterogeneous expression pattern of MAGE-1 antigen in formalin-fixed paraffinembedded lung tumours. Br J Cancer 2000;83:493-497.

31 Schultz-Thater E, Noppen C, Gudat F, et al. NY-ESO-1 tumour associated antigen is a cytoplasmic protein detectable by specific monoclonal antibodies in cell lines and clinical specimens. Br J Cancer 2000;83:204-208.

32 De Smet C, de Backer O, Faraoni I, et al. The activation of human gene $M A G E-1$ in tumor cells is correlated with genome-wide demethylation. Proc Natl Acad Sci USA 1996;93:7149-7153.

33 Van den Eynde BJ, Van der Bruggen P. T cell defined tumor antigens. Curr Opin Immunol 1997;9:684-693.

34 Jungbluth AA, Busam KJ, Kolb D, et al. Expression of MAGE-antigens in normal tissues and cancer. Int J Cancer 2000;85:460-465.

35 Mou DC, Cai SL, Peng JR, et al. Evaluation of MAGE-1 and $M A G E-3$ as tumour-specific markers to detect blood dissemination of hepatocellular carcinoma cells. Br J Cancer 2002;86:110-116.

36 Smith B, Selby P, Southgate J, et al. Detection of melanoma cells in peripheral blood by means of reverse transcription and polymerase chain reaction. Lancet 1991;338:1227-1229.

37 Foss AJ, Guille MJ, Occleston NL, et al. The detection of melanoma cells in peripheral blood by reverse transcription-polymerase chain reaction. Br J Cancer 1995;72:155-159.

38 Hoon DS, Wang Y, Dale PS, et al. Detection of occult melanoma cells in blood with a multiple-marker polymerase chain reaction assay. J Clin Oncol 1995;13: 2109-2116.

39 Fabisiewicz A, Kulik J, Kober P, et al. Detection of circulating breast cancer cells in peripheral blood by a two-marker reverse transcriptase-polymerase chain reaction assay. Acta Biochim Pol. 2004;51:747-755. 